\title{
A Comprehensive Family of Biunivalent Functions Defined by $k$-Fibonacci Numbers
}

\author{
Basem Aref Frasin $\mathbb{D}^{1},{ }^{1}$ Sondekola Rudra Swamy $\mathbb{D}^{2},{ }^{2}$ and Ibtisam Aldawish $\mathbb{D}^{3}$ \\ ${ }^{1}$ Faculty of Science, Department of Mathematics, Al al-Bayt University, Mafraq, Jordan \\ ${ }^{2}$ Department of Computer Science and Engineering, RV College of Engineering, Bengaluru, 560059 Karnataka, India \\ ${ }^{3}$ Department of Mathematics and Statistics, College of Science, Imam Mohammad ibn Saud Islamic University, P.O. Box 90950, \\ Riyadh 11623, Saudi Arabia
}

Correspondence should be addressed to Basem Aref Frasin; bafrasin@yahoo.com

Received 3 September 2021; Accepted 25 September 2021; Published 31 October 2021

Academic Editor: Mohsan Raza

Copyright (c) 2021 Basem Aref Frasin et al. This is an open access article distributed under the Creative Commons Attribution License, which permits unrestricted use, distribution, and reproduction in any medium, provided the original work is properly cited.

By using $k$-Fibonacci numbers, we present a comprehensive family of regular and biunivalent functions of the type $g(z)=$ $z+\sum_{j=2}^{\infty} d_{j} z^{j}$ in the open unit disc $\mathfrak{D}$. We estimate the upper bounds on initial coefficients and also the functional of Fekete-Szegö for functions in this family. We also discuss few interesting observations and provide relevant connections of the result investigated.

\section{Introduction and Notations}

Let $\mathbb{C}$ be the set of all complex numbers and the disc $\{z \in \mathbb{C}:|z|<1\}$ be symbolized by $\mathfrak{D}$. Let $\mathbb{N}=\mathbb{N}_{0} \backslash\{0\}:=$ $\{1,2,3, \cdots\}$ and $\mathbb{R}$ be the collection of all real numbers. We denote the set of all normalized regular functions in $\mathfrak{D}$ that have the series of the form

$$
g(z)=z+\sum_{j=2}^{\infty} d_{j} z^{j}
$$

by $\mathscr{A}$ and the symbol $\delta$ stands for set of all functions of $\mathscr{A}$ that are univalent (or Schlicht) in $\mathfrak{D}$. As per the popular Koebe theorem (see [1]), every function $g \in \mathcal{S}$ has an inverse function given by

$$
\begin{aligned}
g^{-1}(\omega)= & f(\omega)=\omega-d_{2} \omega^{2}+\left(2 d_{2}^{2}-d_{3}\right) \omega^{3} \\
& -\left(5 d_{2}^{3}-5 d_{2} d_{3}+d_{4}\right) \omega^{4}+\cdots,
\end{aligned}
$$

such that $z=g^{-1}(g(z))$ and $\omega=g\left(g^{-1}(\omega)\right),|\omega|<r_{0}(g)$, $r_{0}(g) \geq 1 / 4, z, \omega \in \mathfrak{D}$.

A function $g$ of $\mathscr{A}$ is called biunivalent (or bi-Schlicht) in DD if both $g$ and $g^{-1}$ are univalent (or Schlicht) in $\mathfrak{D}$. Let $\Sigma$ stands for the set of biunivalent (or bi-Schlicht) functions having the form (1). Historically, investigations of the family $\Sigma$ begun five decades ago by Lewin [2] and Brannan and Clunie [3]. Later, Tan [4] found some coefficient estimates for biunivalent functions. In 1986 [5], Brannan and Taha introduced certain well-known subfamilies of $\Sigma$ in $\mathfrak{D}$. Many interesting results related to initial bounds for some special families of $\Sigma$ have appeared in [6-8].

In 2007, the concept of $k$-Fibonacci number sequence $\left\{F_{k, j}\right\}_{j=0}^{\infty}, k \in \mathbb{R}^{+}$was examined by Falcón and Plaza [9] and is given by

$$
\begin{aligned}
F_{k, 0} & =0, \\
F_{k, 1} & =1, \\
F_{k, j+1} & =k F_{k, j}+F_{k, j-1},
\end{aligned}
$$


where $j \in \mathbb{N}$ and

$$
F_{k, j}=\frac{\left(k-t_{k}\right)^{j}-t_{k}^{j}}{\sqrt{k^{2}+4}} \text { with } t_{k}=\frac{k-\sqrt{k^{2}+4}}{2} \text {. }
$$

$F_{1, j}=F_{j}$ is the well-known Fibonacci number sequence. Özgür and Sokól in 2015 [10] proved that if

$$
\tilde{p}_{k}(z)=\frac{1+t_{k}^{2} z^{2}}{1-k t_{k} z-t_{k}^{2} z^{2}},
$$

then,

$$
\begin{aligned}
\tilde{p}_{k}(z) & =1+\left(F_{k, 0}+F_{k, 2}\right) t_{k} z+\left(F_{k, 1}+F_{k, 3}\right) t_{k}^{2} z^{2}+\cdots \\
& =1+k t_{k} z+\left(k^{2}+2\right) t_{k}^{2} z^{2}+\cdots
\end{aligned}
$$

where $t_{k}$ is as in (4) and $z \in \mathfrak{D}$. Further, if $\tilde{p}_{k}(z)=1+\sum \tilde{p}_{k, j} z^{j}$, then, we have

$$
\tilde{p}_{k, j}=t_{k}^{j}\left(F_{k, j-1}+F_{k, j+1}\right), \quad j \in \mathbb{N} .
$$

Fibonacci polynomials, Pell-Lucas polynomials, Gegenbauer polynomials, Chebyshev polynomials, Horadam polynomials, Fermat-Lucas polynomials, and generalizations of them are potentially important in many branches such as architecture, physics, combinatorics, number theory, statistics, and engineering. Additional information is associated with these polynomials one can go through [11-13]. More details about the very popular functional of Fekete-Szegö for biunivalent functions based on $k$-Fibonacci numbers can be found in [14-20].

The recent research trends are the outcomes of the study of functions in $\Sigma$ based on any one of the above-mentioned polynomials, which can be seen in the recent papers [21-28]. Generally, interest was shown to estimate the first two coefficient bounds and the functional of Fekete-Szegö for some subfamilies of $\Sigma$.

For functions $g$ and $f$ regular in $\mathfrak{D}, g$ is said to subordinate $f$, if there is a Schwarz function $\psi$ in $\mathfrak{D}$, such that $\psi(0)=0,|\psi(z)|<1$, and $g(z)=f(\psi(z)), z \in \mathfrak{D}$. This subordination is indicated as $g \prec f$. In particular, if $f \in \mathcal{S}$, then $g(z) \prec f(z) \Leftarrow g(0)=f(0)$ and $g(\mathfrak{D}) \subset f(\mathfrak{D})$.

Inspired by the recent articles and the new trends on functions in $\Sigma$, we present a comprehensive family of $\Sigma$ defined by using $k$-Fibonacci numbers as given by (3) with $F_{k, j}$ as in (4).

Throughout this paper, $g^{-1}(\omega)=f(\omega)$ is as in (2), $T_{k}=$ $k-\left(k^{2}+2\right) t_{k}, t_{k}$ is as in (4), and $\tilde{p}_{k}$ is as in (5).
Definition 1. A function $g \in \Sigma$ having the power series (1) is said to be in the family $\operatorname{SR} \widetilde{S}_{\Sigma}^{\tau}\left(\gamma, \mu, \tilde{p}_{k}\right)$, if

$$
\begin{aligned}
& \frac{z\left(g^{\prime}(z)\right)^{\tau}+\mu z^{2} g^{\prime \prime}(z)}{\gamma g(z)+(1-\gamma) z} \prec \tilde{p}_{k}(z), \quad z \in \mathfrak{D} \\
& \frac{\omega\left(g^{\prime}(\omega)\right)^{\tau}+\mu z^{2} g^{\prime \prime}(\omega)}{\gamma g(\omega)+(1-\gamma) \omega} \prec \tilde{p}_{k}(\omega), \quad \omega \in \mathfrak{D},
\end{aligned}
$$

where $\tau \geq 1,0 \leq \gamma \leq 1$, and $\mu \geq 0$.

Remark 2. The function families $\operatorname{SR} \mathfrak{S}_{\Sigma}^{\tau}\left(\gamma, 0, \tilde{p}_{k}\right)$ and $\operatorname{SR} \widetilde{S}_{\Sigma}^{1}(\gamma$, $\left.\mu, \tilde{p}_{k}\right)$ were investigated by Frasin et al. [29].

It is interesting to note that (i) $\gamma=1$, (ii) $\gamma=0$, and (iii) $\mu=1$ lead the family $\operatorname{SR} \widetilde{\Xi}_{\Sigma}^{\tau}\left(\gamma, \mu, \tilde{p}_{k}\right)$ to various subfamilies, as illustrated in the following:

(1) $\operatorname{SR}_{\Sigma}^{\tau}\left(1, \mu, \tilde{p}_{k}\right) \equiv L_{\Sigma}^{\tau}\left(\mu, \tilde{p}_{k}\right)$ is the family of functions $g \in \sum$ satisfying

$$
\begin{aligned}
& \frac{z\left(g^{\prime}(z)\right)^{\tau}}{g(z)}+\mu\left(\frac{z^{2} g^{\prime \prime}(z)}{g(z)}\right) \prec \tilde{p}_{k}(z) \text { and } \frac{\omega\left(f^{\prime}(\omega)\right)^{\tau}}{f(\omega)} \\
& +\mu\left(\frac{\omega^{2} f^{\prime \prime}(\omega)}{f(\omega)}\right) \prec \tilde{p}_{k}(\omega), \quad z, \omega \in \mathfrak{D}
\end{aligned}
$$

(2) $\operatorname{SR}_{\Sigma}^{\tau}\left(0, \mu, \tilde{p}_{k}\right) \equiv K_{\Sigma}^{\tau}\left(\mu, \tilde{p}_{k}\right)$ is the set of functions $g \in \Sigma$ satisfying

$$
\begin{gathered}
\left(g^{\prime}(z)^{\tau}+\mu z g^{\prime \prime}(z) \prec \tilde{p}_{k}(z) \text { and }\left(f^{\prime}(\omega)\right)^{\tau}\right. \\
+\mu \omega f^{\prime \prime}(\omega) \prec \tilde{p}_{k}(\omega), \quad z, \omega \in \mathfrak{D}
\end{gathered}
$$

(3) $\operatorname{SR}_{\Sigma}^{\tau}\left(\gamma, 1, \tilde{p}_{k}\right) \equiv M_{\Sigma}^{\tau}\left(\gamma, \tilde{p}_{k}\right)$ is the collection of functions $g \in \Sigma$ satisfying

$$
\begin{aligned}
& \frac{z\left(g^{\prime}(z)\right)^{\tau}+z^{2} g^{\prime \prime}(z)}{\gamma g(z)+(1-\gamma) z} \prec \tilde{p}_{k}(z), \quad z \in \mathfrak{D}, \\
& \frac{\omega\left(f^{\prime}(\omega)\right)^{\tau}+\omega^{2} f^{\prime \prime}(\omega)}{\gamma f(\omega)+(1-\gamma) \omega} \prec \tilde{p}_{k}(\omega), \quad \omega \in \mathfrak{D}
\end{aligned}
$$

Remark 3. We note that (i) $L_{\Sigma}^{\tau}\left(1, \tilde{p}_{k}\right) \equiv M_{\Sigma}^{\tau}\left(1, \tilde{p}_{k}\right)$ and (ii) $K_{\Sigma}^{\tau}\left(1, \tilde{p}_{k}\right) \equiv M_{\Sigma}^{\tau}\left(0, \tilde{p}_{k}\right)$. 
Remark 4.

(i) When $\tau=1$, the family $K_{\Sigma}^{1}\left(\mu, \tilde{p}_{k}\right)$ was introduced by Frasin et al. [30]

(ii) The family $\left.L_{\Sigma}^{1}\left(0, \tilde{p}_{k}\right) \equiv \mathcal{S}_{\Sigma}^{*}\left(\tilde{p}_{k}\right)\right)$ was mentioned by Güney et al. [18], when $\mu=0$ and $\tau=1$

(iii) For $\mu=0$ and $k=1$, the class $L_{\Sigma}^{\tau}\left(0, \tilde{p}_{1}\right) \equiv \mathcal{S}_{\Sigma}\left(\tilde{p}_{1}\right)$ was investigated by Magesh et al. [31]

We now state the following lemma, which we will be using in the proof of our theorem.

Lemma 5 (see [32]). If $p \in P$, where $P$ is the collection of regular functions $p$ in $\mathfrak{D}$, satisfying $\mathfrak{R}(p(z))>0, z \in \mathfrak{D}$, with $p(z)=1+p_{1} z+p_{2} z^{2}+\cdots, z \in \mathfrak{D}$, then $\left|p_{i}\right| \leq 2$, for each $i$.

In the next section, we derive the estimates for $\left|d_{2}\right|,\left|d_{3}\right|$ and obtain the Fekete-Szegö [33] inequalities for functions in the class $\operatorname{SR} \widetilde{\Im}_{\Sigma}^{\tau}\left(\gamma, \mu, \tilde{p}_{k}\right)$.

\section{Coefficient Bounds and Fekete- Szegö Functional}

In this section, we offer to get the upper bounds on initial coefficients and find the functional of Fekete-Szegö for functions $\in \operatorname{SR} \widetilde{S}_{\Sigma}^{\tau}\left(\gamma, \mu, \tilde{p}_{k}\right)$.

Theorem 6. Let $\tau \geq 1,0 \leq \gamma \leq 1$, and $\mu \geq 0$. If $g(z)$ of the form (1) in the family $\operatorname{SR}_{\Sigma}^{\tau}\left(\gamma, \mu, \tilde{p}_{k}\right)$, then

$$
\left|d_{2}\right| \leq \frac{k \sqrt{k}\left|t_{k}\right|}{\sqrt{\left|\left(\gamma^{2}+(\tau-\gamma)(2 \tau+1)+2 \mu(3-\gamma)\right) k^{2} t_{k}+(2(\mu+\tau)-\gamma)^{2} T_{k}\right|}},
$$

$$
\begin{aligned}
\left|d_{3}\right| & \leq \frac{k\left|t_{k}\right|}{3(2 \mu+\tau)-\gamma} \\
& +\frac{k^{3} t_{k}^{2}}{\left|\left(\gamma^{2}+(\tau-\gamma)(2 \tau+1)+2 \mu(3-\gamma)\right) k^{2} t_{k}+(2(\mu+\tau)-\gamma)^{2} T_{k}\right|}
\end{aligned}
$$

and for $\delta \in \mathbb{R}$,

$$
\left|d_{3}-\delta d_{2}^{2}\right| \leq \begin{cases}\frac{k\left|t_{k}\right|}{3(2 \mu+\tau)-\gamma} & ;|1-\delta| \leq J, \\ \frac{k^{3} t_{k}^{2}|1-\delta|}{\left|\left(\gamma^{2}+(\tau-\gamma)(2 \tau+1)+2 \mu(3-\gamma)\right) k^{2} t_{k}+(2(\mu+\tau)-\gamma)^{2} T_{k}\right|} & ;|1-\delta| \geq J,\end{cases}
$$

where

$$
\begin{aligned}
J= & \frac{1}{3(2 \mu+\tau)-\gamma} \\
& \cdot\left[\gamma^{2}+(\tau-\gamma)(2 \tau+1)+2 \mu(3-\gamma)+(2(\mu+\tau)-\gamma)^{2}\left|\frac{T_{k}}{k^{2} t_{k}}\right|\right] .
\end{aligned}
$$

Proof. Let the function $g \in S R \widetilde{S}_{\Sigma}^{\tau}\left(\gamma, \mu, \tilde{p}_{k}\right)$. Then, from Definition 1 , we have

$$
\begin{gathered}
\frac{z\left(g^{\prime}(z)\right)^{\tau}+\mu z^{2} g^{\prime \prime}(z)}{\gamma g(z)+(1-\gamma) z} \prec \tilde{p}_{k}(u(z)), \quad z \in \mathfrak{D}, \\
\frac{\omega\left(f^{\prime}(\omega)\right)^{\tau}+\mu \omega^{2} f^{\prime \prime}(\omega)}{\gamma f(\omega)+(1-\gamma) \omega} \prec \tilde{p}_{k}(v(\omega)), \quad \omega \in \mathfrak{D} .
\end{gathered}
$$

Let $p(z)=1+p_{1} z+p_{2} z^{2}+\cdots$, and $p \prec \tilde{p}_{k}$. Then, there exists a regular function $u$ with $|u(z)|<1$ in $\mathfrak{D}$ and $p(z)=$ $\tilde{p}_{k}(u(z))$. Therefore, the function $m(z)$ is in the class $P$, where

$$
m(z)=\frac{1+u(z)}{1-u(z)}=1+u_{1} z+u_{2} z^{2}+\cdots
$$

So it follows that

$u(z)=\frac{m(z)-1}{m(z)+1}=\frac{u_{1}}{2} z+\left(u_{2}-\frac{u_{1}^{2}}{2}\right) \frac{z^{2}}{2}+\left(u_{3}-u_{1} u_{2}+\frac{u_{1}^{3}}{4}\right) \frac{z^{3}}{2}+\cdots$,

$$
\begin{aligned}
\tilde{p}_{k}(u(z))= & 1+\tilde{p}_{k, 1}\left(\frac{u_{1} z}{2}+\left(u_{2}-\frac{u_{1}^{2}}{2}\right) \frac{z^{2}}{2}+\cdots\right) \\
& +\tilde{p}_{k, 2}\left(\frac{u_{1} z}{2}+\left(u_{2}-\frac{u_{1}^{2}}{2}\right) \frac{z^{2}}{2}+\cdots\right)^{2}+\cdots \\
= & 1+\frac{\tilde{p}_{k, 1} u_{1} z}{2}+\left(\frac{1}{2}\left(u_{2}-\frac{u_{1}^{2}}{2}\right) \tilde{p}_{k, 1}+\frac{u_{1}^{2}}{4} \tilde{p}_{k, 2}\right) z^{2}+\cdots
\end{aligned}
$$


Similarly, it follows that

$$
\tilde{p}_{k}(v(\omega))=1+\frac{\tilde{p}_{k, 1} v_{1} \omega}{2}+\left(\frac{1}{2}\left(v_{2}-\frac{v_{1}^{2}}{2}\right) \tilde{p}_{k, 1}+\frac{v_{1}^{2}}{4} \tilde{p}_{k, 2}\right) \omega^{2}+\cdots,
$$

where $v$ is a regular function such that $|v(\omega)|<1$ in $\mathfrak{D}$ such that $p(\omega)=\tilde{p}_{k}(v(\omega))$ and the function $l(\omega)$ is in the class $P$, where

$$
l(\omega)=\frac{1+v(\omega)}{1-v(\omega)}=1+v_{1} \omega+v_{2} \omega^{2}+\cdots
$$

By virtue of (14), (15), (18), and (19), we obtain

$$
\begin{gathered}
(2(\mu+\tau)-\gamma) d_{2}=\frac{u_{1} k t_{k}}{2} \\
(3(2 \mu+\tau)-\gamma) d_{3}+\left(\gamma^{2}-2 \gamma(\mu+\tau)+2 \tau(\tau-1)\right) d_{2}^{2} \\
=\frac{1}{2}\left(u_{2}-\frac{u_{1}^{2}}{2}\right) k t_{k}+\frac{u_{1}^{2}}{4}\left(k^{2}+2\right) t_{k}^{2}, \\
-(2(\mu+\tau)-\gamma) d_{2}=\frac{v_{1} k t_{k}}{2}, \\
(3(2 \mu+\tau)-\gamma)\left(2 d_{2}^{2}-d_{3}\right)+\left(\gamma^{2}-2 \gamma(\mu+\tau)+2 \tau(\tau-1)\right) d_{2}^{2} \\
=\frac{1}{2}\left(v_{2}-\frac{v_{1}^{2}}{2}\right) k t_{k}+\frac{v_{1}^{2}}{4}\left(k^{2}+2\right) t_{k}^{2} .
\end{gathered}
$$

From (21) and (23), we get

$$
u_{1}=-v_{1}
$$

and also,

$$
2(2(\mu+\tau)-\gamma)^{2} d_{2}^{2}=\frac{\left(u_{1}^{2}+v_{1}^{2}\right) k^{2} t_{k}^{2}}{4} .
$$

If we add (26) and (24), then we obtain

$$
\begin{aligned}
& 2\left(\gamma^{2}+(\tau-\gamma)(2 \tau+1)+2 \mu(3-\gamma)\right) d_{2}^{2}=\frac{1}{2}\left(u_{2}+v_{2}\right) k t_{k} \\
& \quad-\frac{1}{4}\left(k t_{k}-\left(k^{2}+2\right) t_{k}^{2}\right)\left(u_{1}^{2}+v_{1}^{2}\right) .
\end{aligned}
$$

Substituting the value of $\left(u_{1}^{2}+v_{1}^{2}\right)$ from (26) in (27), we get

$$
d_{2}^{2}=\frac{k^{3} t_{k}^{2}\left(u_{2}+v_{2}\right)}{\left.4\left[\gamma^{2}+(\tau-\gamma)(2 \tau+1)+2 \mu(3-\gamma)\right) k^{2} t_{k}+(2(\mu+\tau)-\gamma)^{2} T_{k}\right]},
$$

which gets (10), on using Lemma 5 .

On using (25) in the subtraction of (24) from (26), we arrive at

$$
d_{3}=d_{2}^{2}+\frac{k t_{k}\left(u_{2}-v_{2}\right)}{4(3(2 \mu+\tau)-\gamma)}
$$

Then, in view of Lemma 5 and equation (28), (29) reduces to (11).

From (28) and (29), for $\delta \in \mathbb{R}$, we can easily compute that

$$
\begin{aligned}
\left|d_{3}-\delta d_{2}^{2}\right|= & k\left|t_{k}\right| \mid\left(T(\delta)+\frac{1}{4(3(2 \mu+\tau)-\gamma)}\right) u_{2} \\
& +\left(T(\delta)-\frac{1}{4(3(2 \mu+\tau)-\gamma)}\right) v_{2} \mid
\end{aligned}
$$

where

$$
T(\delta)=\frac{(1-\delta) k^{2} t_{k}}{4\left[\left(\gamma^{2}+(\tau-\gamma)(2 \tau+1)+2 \mu(3-\gamma)\right) k^{2} t_{k}+(2(\mu+\tau)-\gamma)^{2} T_{k}\right]} .
$$

In view of (4), we find that

$$
\left|d_{3}-\delta d_{2}^{2}\right| \leq \begin{cases}\frac{k\left|t_{k}\right|}{(3(2 \mu+\tau)-\gamma)} & ; 0 \leq|T(\delta)| \leq \frac{1}{4(3(2 \mu+\tau)-\gamma)}, \\ 4 k\left|t_{k}\right||T(\delta)| & ;|T(\delta)| \geq \frac{1}{4(3(2 \mu+\tau)-\gamma)},\end{cases}
$$

which enable us to conclude (12) with $J$ as in (13). Theorem 6 is proved.

Remark 7 . By taking $\tau=1$ in the above theorem, we obtain a result of Frasin et al. ([29], Corollary 3.4) and if we let $\mu=0$ in the above theorem, we get another result of Frasin et al. ([29], Corollary 3.7).

Remark 8. Allowing $k=\gamma=1$ and $\mu=0$ in the above theorem, we have Theorem 2.3 of Magesh et al. [31].

Remark 9. Letting $\tau=\gamma=1$ and $\mu=0$ in the Theorem 6, we obtain two results of Güney et al. ([18], Corollary 10 and Corollary 23). Further, if we take $k=1$, we get results of Güney et al. ([17], Corollary 1 and Corollary 4).

In Section 3, few interesting consequences and relevant observations of the main result are mentioned. 


\section{Outcome of the Main Result}

By setting (i) $\gamma=1$, (ii) $\gamma=0$, and (iii) $\mu=1$ in our main theorem, we obtain the following results, respectively.

Corollary 10. If the function $g \in L_{\Sigma}^{\tau}\left(\mu, \tilde{p}_{k}\right)$, then

$$
\begin{aligned}
& \left|d_{2}\right| \leq \frac{k \sqrt{k}\left|t_{k}\right|}{\sqrt{\left|(\tau(2 \tau-1)+4 \mu) k^{2} t_{k}+(2(\mu+\tau)-1)^{2} T_{k}\right|}}, \\
& \left|d_{3}\right| \leq \frac{k\left|t_{k}\right|}{3(2 \mu+\tau)-1}+\frac{k^{3} t_{k}^{2}}{\left|(\tau(2 \tau-1)+4 \mu) k^{2} t_{k}+(2(\mu+\tau)-1)^{2} T_{k}\right|},
\end{aligned}
$$

and for $\delta \in \mathbb{R}$,

$$
\left|d_{3}-\delta d_{2}^{2}\right| \leq \begin{cases}\frac{k\left|t_{k}\right|}{3(2 \mu+\tau)-1} & ;|1-\delta| \leq J_{1}, \\ \frac{k^{3} t_{k}^{2}|1-\delta|}{\left|(\tau(2 \tau-1)+4 \mu) k^{2} t_{k}+(2(\mu+\tau)-1)^{2} T_{k}\right|} & ;|1-\delta| \geq J_{1},\end{cases}
$$

where

$$
J_{1}=\frac{1}{3(2 \mu+\tau)-1}\left(\tau(2 \tau-1)+4 \mu+(2(\mu+\tau)-1)^{2}\left|\frac{T_{k}}{k^{2} t_{k}}\right|\right) \text {. }
$$

\section{Remark 11.}

(i) By taking $\mu=0$ and $k=1$ in the above corollary, we obtain Theorem 2.3 of Magesh et al. [31]

(ii) By allowing $\mu=0$ and $\tau=1$ in the above corollary, we get two results Güney et al. ([18], Corollary 10 and Corollary 23)
Corollary 12. If the function $g \in K_{\Sigma}^{\tau}\left(\mu, \tilde{p}_{k}\right)$, then

$$
\begin{aligned}
& \left|d_{2}\right| \leq \frac{k \sqrt{k}\left|t_{k}\right|}{\sqrt{\left|(\tau(2 \tau+1)+6 \mu) k^{2} t_{k}+4(\mu+\tau)^{2} T_{k}\right|}}, \\
& \left|d_{3}\right| \leq \frac{k^{3} t_{k}^{2}}{\left|(\tau(2 \tau+1)+6 \mu) k^{2} t_{k}+4(\mu+\tau)^{2} T_{k}\right|}+\frac{k\left|t_{k}\right|}{3(2 \mu+\tau)},
\end{aligned}
$$

and for some $\delta \in \mathbb{R}$,

$$
\left|d_{3}-\delta d_{2}^{2}\right| \leq \begin{cases}\frac{k\left|t_{k}\right|}{3(2 \mu+\tau)} & ;|1-\delta| \leq J_{2} \\ \frac{k^{3} t_{k}^{2}|1-\delta|}{\left|(\tau(2 \tau+1)+6 \mu) k^{2} t_{k}+4(\mu+\tau)^{2} T_{k}\right|} & ;|1-\delta| \geq J_{2}\end{cases}
$$

where

$$
J_{2}=\frac{1}{3(2 \mu+\tau)}\left[\tau(2 \tau+1)+6 \mu+4\left(\mu+\tau^{2}\right)\left|\frac{T_{k}}{k^{2} t_{k}^{2}}\right|\right] .
$$

Remark 13. For $\tau=1$, Corollary 12 reduces to a result of Frasin et al. ([30], Corollary 3.6). Further, allowing $k=1$, we get Corollary 10 of Altnkaya [22].

Corollary 14. If the function $g \in M_{\Sigma}^{\tau}\left(\gamma, \tilde{p}_{k}\right)$, then

$$
\begin{aligned}
& \left|d_{2}\right| \leq \frac{k \sqrt{k}\left|t_{k}\right|}{\sqrt{\mid\left((1-\gamma)^{2}+(\tau-\gamma)(2 \tau+1)+5-\gamma\right) k^{2} t_{k}+(2(1+\tau)-\gamma)^{2} T_{k}} \mid}, \\
& \left|d_{3}\right| \leq \frac{k\left|t_{k}\right|}{3(2+\tau)-\gamma}+\frac{k^{3} t_{k}^{2}}{\left|(4 \mu+1) k^{2} t_{k}+(2 \mu+1)^{2} T_{k}\right|},
\end{aligned}
$$

and for $\delta \in \mathbb{R}$,

$$
\left|d_{3}-\delta d_{2}^{2}\right| \leq \begin{cases}\frac{k\left|t_{k}\right|}{3(2+\tau)-\gamma} & ;|1-\delta| \leq J_{3}, \\ \frac{k^{3} t_{k}^{2}|1-\delta|}{\left|\left((1-\gamma)^{2}+(\tau-\gamma)(2 \tau+1)+5-\gamma\right) k^{2} t_{k}+(2(1+\tau)-\gamma)^{2} T_{k}\right|} & ;|1-\delta| \geq J_{3},\end{cases}
$$

where

$$
J_{3}=\frac{1}{3(2+\tau)-\gamma}\left(\left((1-\gamma)^{2}+(\tau-\gamma)(2 \tau+1)+5-\gamma\right)+(2(1+\tau)-\gamma)^{2}\left|\frac{T_{k}}{k^{2} t_{k}}\right|\right) .
$$




\section{Conclusion}

A comprehensive family of biunivalent (or bi-Schlicht) functions is introduced by using $k$-Fibonacci numbers. Bounds of the first two coefficients $\left|d_{2}\right|$ and $\left|d_{3}\right|$ and the celebrated Fekete-Szegö functional have been fixed for this family. Through corollaries of our main result, we have highlighted many interesting new consequences.

A comprehensive family examined in this research paper could inspire further research related to other aspects such as a comprehensive family using $q$-derivative operator, a meromorphic biunivalent function family associated with Al-Oboudi differential operator, and a comprehensive family using integrodifferential operator.

\section{Data Availability}

There is no data used for this manuscript.

\section{Conflicts of Interest}

The authors confirm that there are no competing interests regarding the publication of this manuscript.

\section{Authors' Contributions}

The authors contributed equally in the preparation of this manuscript and have approved the final version of the manuscript.

\section{References}

[1] P. L. Duren, Univalent Functions, Grundlehren der Mathematischen Wissenschaften, Band 259, Springer-Verlag, New York, 1983.

[2] M. Lewin, "On a coefficient problem for bi-univalent functions," Proceedings of the American Mathematical Society, vol. 18, no. 1, pp. 63-68, 1967.

[3] D. A. Brannan and J. G. Clunie, Aspects of Contemporary Complex Analysis, Proceedings of the NATO Advanced Study Institute Held at University of Durhary, Academic press, Newyork, 1979.

[4] D. L. Tan, "Coefficient estimates for bi-univalent functions," Chinese Annals of Mathematics, Series A, vol. 5, pp. 559-568, 1984.

[5] D. A. Brannan and T. S. Taha, "On some classes of biunivalent functions," Mathematical Analysis and Its Applications. Kuwait, pp 53-60, KFAS Proceedings Series, Vol. 3 (1985), S. M. Mazhar, A. Hamoui, and N. S. Faour, Eds., Pergamon Press (Elsevier Science Limited), Oxford, 1988, see also Studia Univ. Babes-Bolyai Math., 31(2) (1986), 70-77.

[6] B. A. Frasin, "Coefficient bounds for certain classes of biunivalent functions," Hacettepe Journal of Mathematics and Statistics, vol. 43, no. 3, pp. 383-389, 2014.

[7] B. A. Frasin and M. K. Aouf, "New subclasses of bi-univalent functions," Applied Mathematics Letters, vol. 24, no. 9, pp. 1569-1573, 2011.

[8] H. M. Srivastava, A. K. Mishra, and P. Gochhayat, "Certain subclasses of analytic and bi-univalent functions," Applied Mathematics Letters, vol. 23, no. 10, pp. 1188-1192, 2010.
[9] S. Falcón and A. Plaza, "On the Fibonacci_k_ -numbers," Chaos, Solitons \& Fractals, vol. 32, no. 5, pp. 1615-1624, 2007.

[10] N. Y. Özgür and J. Sokół, "On starlike functions connected with $k$-Fibonacci numbers," Bulletin of the Malaysian Mathematical Sciences Society, vol. 38, no. 1, pp. 249-258, 2015.

[11] P. Filipponi and A. F. Horadam, "Derivative sequences of Fibonacci and Lucas polynomials," Applications of Fibonacci Numbers, vol 4 (1990), pp 99-108, Proceedings of the Fourth International Conference on Fibonacci Numbers and their Applications, , no. 1991, pp. 99-108, Wake Forest University, Winston-Salem, North Carolina; Springer (Kluwer Academic Publishers), Dordrecht, Boston and London.

[12] P. Filipponi and A. F. Horadam, "Second derivative sequence of Fibonacci and Lucas polynomials," The Fibonacci Quarterly, vol. 31, pp. 194-204, 1993.

[13] T.-T. Wang and W.-P. Zhang, "Some identities involving Fibonacci, Lucas polynomials and their applications," Bulletin mathématique de la société des sciences mathématiques de Roumanie, vol. 55, no. 103, pp. 95-103, 2012.

[14] J. Dziok, R. K. Raina, and J. Sokół, “Certain results for a class of convex functions related to a shell-like curve connected with Fibonacci numbers," Computers \& Mathematics with Applications, vol. 61, no. 9, pp. 2605-2613, 2011.

[15] J. Dziok, R. K. Raina, and J. Sokól, “On $\alpha$-convex functions related to shell-like functions connected with Fibonacci numbers," Applied Mathematics and Computation, vol. 218, no. 3, pp. 996-1002, 2011.

[16] H. Ö. Güney, "Coefficient bounds for analytic bi-Bazilevič functions related to shell-like curves connected with Fibonacci numbers," Sahand Communications in Mathematical Analysis, vol. 16, no. 1, pp. 149-160, 2019.

[17] H. Ö. Güney, G. Murugusundaramoorthy, and J. Sokól, "Subclasses of bi-univalent functions related to shell-like curves connected with Fibonacci numbers," Acta Universitatis Sapientiae, Mathematica, vol. 10, no. 1, pp. 70-84, 2018.

[18] H. Ö. Güney, G. Murugusundaramoorthy, and J. Sokól, “Certain subclasses of bi-univalent functions related to k-Fibonacci numbers," Communications, vol. 68, no. 2, pp. 1909-1921, 2019.

[19] R. K. Raina and J. Sokól, "Fekete-Szegö problem for some starlike functions related to shell-like curves," Mathematica Slovaca, vol. 66, no. 1, pp. 135-140, 2016.

[20] J. Sokól, R. K. Raina, and N. Yilmaz Özgür, “Applications of Fibonacci numbers for the starlike analytic functions," Hacettepe Journal of Mathematics and Statistics, vol. 1, no. 1036, pp. 121-127, 2015.

[21] I. Aldawish, T. Al-Hawary, and B. A. Frasin, "Subclasses of biunivalent functions defined by Frasin differential operator," Mathematics, vol. 8, no. 5, p. 783, 2020.

[22] S. Altnkaya, "Bounds for a new subclass of bi-univalent functions subordinate to the Fibonacci numbers," Turkish Journal of Mathematics, vol. 44, no. 2, pp. 553-560, 2020.

[23] A. Amourah, B. A. Frasin, and T. Abdeljawad, "Fekete-Szegö inequality for analytic and biunivalent functions subordinate to Gegenbauer polynomials," Journal of Function Spaces, vol. 2021, Article ID 5574673, 7 pages, 2021.

[24] S. M. El-Deeb, T. Bulboača, and B. M. El-Matary, "Maclaurin coefficient estimates of bi-univalent functions connected with the q-derivative," Mathematics, vol. 8, no. 3, p. 418, 2020.

[25] H. M. Srivastava, Ş. Altınkaya, and S. Yalçın, "Certain subclasses of bi-univalent functions associated with the Horadam 
polynomials," Iranian journal of science and technology, transactions A: Science, vol. 43, no. 4, pp. 1873-1879, 2019.

[26] S. R. Swamy, "Coefficient bounds for Al-Oboudi type biunivalent functions based on a modified sigmoid activation function and Horadam polynomials," Earthline Journal of Mathematical Sciences, vol. 7, no. 2, pp. 251-270, 2021.

[27] S. R. Swamy, S. Bulut, and Y. Sailaja, "Some special families of holomorphic and Salagean type bi-univalent functions associated with Horadam polynomials involving modified sigmoid activation function," Hacettepe Journal of Mathematics and Statistics, vol. 50, no. 3, pp. 710-720, 2021.

[28] S. R. Swamy, A. K. Wanas, and Y. Sailaja, "Some special families of holomorphic and Sălăgean type bi-univalent functions associated with (m,n)-Lucas polynomials," Communications in Mathematics and Applications, vol. 11, no. 4, pp. 1-12, 2020.

[29] B. A. Frasin, S. R. Swamy, and Y. Sailaja, "Coefficient bounds for Al-Oboudi type bi-univalent functions connected with a modified sigmoid activated function and $\mathrm{k}$-Fibonacci numbers (preprint)".

[30] B. A. Frasin, S. R. Swamy, and J. Nirmala, "Some special families of holomorphic and Al-Oboudi type bi-univalent functions related to $\mathrm{k}$-Fibonacci numbers involving modified sigmoid activation function," Afrika Matematika, vol. 32, no. 3-4, pp. 631-643, 2021.

[31] N. Magesh, V. K. Balaji, and C. Abirami, "Certain classes of biunivalent functions related to Shell-like curves connected with Fibonacci numbers," https://arxiv.org/abs/1810.06216.

[32] C. Pommerenke, Univalent Functions, Vandenhoeck and Ruprecht, Göttingen, 1975.

[33] M. Fekete and G. Szegö, "Eine Bemerkung Über Ungerade Schlichte Funktionen," Journal of the London Mathematical Society, vol. s1-8, no. 2, pp. 85-89, 1933. 